Homology, Homotopy and Applications, vol.20(1), 2018, pp.237-250

\title{
CYCLIC VS MIXED HOMOLOGY
}

\section{ULRICH KRÄHMER AND DYLAN MADDEN}

\author{
(communicated by Jonathan M. Rosenberg)
}

\begin{abstract}
The spectral theory of the Karoubi operator due to Cuntz and Quillen is extended to general mixed (duchain) complexes, that is, chain complexes which are also cochain complexes. Connes' coboundary map $B$ can be viewed as a perturbation of the noncommutative De Rham differential by a polynomial in the Karoubi operator. The homological impact of such perturbations is expressed in terms of two short exact sequences.
\end{abstract}

\section{Introduction and overview}

\subsection{Mixed complexes}

Inspired by Connes' work on cyclic homology $[\mathbf{2}, \mathbf{3}]$, Dwyer and Kan $[\mathbf{7}, \mathbf{8}]$ initiated the study of general chain complexes which simultaneously are cochain complexes:

Definition 1.1. A mixed complex of $R$-modules is a triple $(\Omega, b, d)$ where $(\Omega, b)$ and $(\Omega, d)$ are a chain respectively a cochain complex:

$$
\cdots \underset{d}{\stackrel{b}{\gtrless}} \Omega_{2} \underset{d}{\stackrel{b}{\gtrless}} \Omega_{1} \underset{d}{\stackrel{b}{\gtrless}} \Omega_{0} \underset{0}{\stackrel{0}{<}} 0, \quad d^{2}=b^{2}=0 .
$$

The mixed homology $\mathrm{H}(\Omega, b, d)$ is the homology of $(\mathrm{T}(\Omega), b+d)$, where

$$
\mathrm{T}_{n}(\Omega):=\bigoplus_{i \geqslant 0} \hat{\Omega}_{n-2 i}, \quad \hat{\Omega}_{i}:=\Omega_{i} / \operatorname{im} \xi, \quad \xi:=b d+d b .
$$

Dwyer and Kan used the term duchain rather than mixed complex, but the latter (introduced by Kassel [12]) is now the standard terminology, although it is mostly associated with the special case $\xi=0$.

The motivating examples are the noncommutative differential forms over an associative algebra with the De Rham differential $d$ and the Hochschild boundary map $b$, see Section 1.4 and Example 2.8 below, or [15, Section 2.6] for a detailed account. However, mixed complexes appear in a wide range of contexts, e.g., Poisson manifolds $[\mathbf{1}, \mathbf{1 3}]$, Lie-Rinehart algebras (Lie algebroids) $[\mathbf{1 1}]$, and Hopf algebras $[\mathbf{4 , 5}, \mathbf{1 0}]$.

U.K. thanks Gabriella Böhm, Niels Kowalzig and Tomasz Maszczyk for discussions, and IMPAN Warsaw and the Wigner Institute Budapest for hospitality. Both authors thank the referee for a very careful reading of the manuscript that lead to numerous improvements.

Received May 18, 2017, revised August 18, 2017; published on February 14, 2018.

2010 Mathematics Subject Classification: 19D55, 18G60, 18G30, 18 G35.

Key words and phrases: mixed complex, cyclic homology, mixed homology, noncommutative differential form.

Article available at http://dx.doi.org/10.4310/HHA.2018.v20.n1.a14

Copyright (c) 2018, International Press. Permission to copy for private use granted. 


\subsection{The spectral decomposition}

Our aim here is to revisit the construction of cyclic homology from the perspective of general mixed complexes. To this end, we view $\Omega$ as a $k[x]$-module, where $k$ is the centre of $R$ and $x$ acts by $\xi$. Thus $\Omega$ defines a sheaf of mixed complexes over the affine line $k$; this generalises the spectral decomposition of $\Omega$ considered by Cuntz and Quillen [6].

The localisation $S^{-1} \Omega:=k\left[x, x^{-1}\right] \otimes_{k[x]} \Omega$ is contractible as a chain and cochain complex, for if $\xi$ is invertible, then we have

$$
b\left(\xi^{-1} d\right)+\left(\xi^{-1} d\right) b=\mathrm{id}, \quad d\left(\xi^{-1} b\right)+\left(\xi^{-1} b\right) d=\mathrm{id} .
$$

Thus, the only stalk of $\Omega$ supporting (co)homology is $\hat{\Omega}=\Omega /$ im $\xi$ at $x=0$. A particularly well-behaved class of mixed complexes is therefore formed by those which are globally contractible to $\hat{\Omega}$ :

Definition 1.2. We call $(\Omega, b, d)$ a (co)homological skyscraper if

$$
\Omega \rightarrow \hat{\Omega}=\Omega / \operatorname{im} \xi
$$

is a quasiisomorphism of (co)chain complexes.

This holds for example when $\Omega=\operatorname{ker} \xi \oplus \operatorname{im} \xi$ so that $\operatorname{im} \xi \cong S^{-1} \Omega$, and in particular, when $k$ is a field and $\xi$ is diagonalisable over $k$.

Example 1.3. For an example of a non-skyscraper, define

$$
\Omega_{n}:=\left\{\begin{array}{llll}
R \oplus R & n=0,1, & d: \Omega_{0} \rightarrow \Omega_{1}, & (r, s) \mapsto(r, s), \\
0 & n>1, & b: \Omega_{1} \rightarrow \Omega_{0}, & (u, v) \mapsto(0, u) .
\end{array}\right.
$$

The homology of $\Omega$ is $R$ in both degrees and so is that of $\hat{\Omega}$, but while the map induced on homology by the quotient $\Omega \rightarrow \hat{\Omega}$ is the identity in degree 0 it vanishes in degree 1 , so $\Omega$ is not a homological skyscraper.

We will provide further toy examples that illustrate the definitions and results throughout the text. As a first example of real interest, we mention:

Example 1.4. Consider the De Rham complex $(\Omega, d)$ of a compact oriented Riemannian manifold, and let $b$ be the adjoint of $d$ with respect to the Riemannian volume form. Then $\xi$ is the Laplace operator and the spectral decomposition of this elliptic (essentially) self-adjoint operator yields $\Omega=\operatorname{ker} \xi \oplus \operatorname{im} \xi$, so $\Omega$ is a skyscraper and is contractible to $\operatorname{ker} \xi$, the space of harmonic forms. The results of this paper can therefore also be viewed as an abstraction of the Hodge theorem.

\subsection{Statement of the main results}

The noncommutative differential forms over an algebra are not a skyscraper with respect to the De Rham differential $d$, see Example 2.18, but they are with respect to the coboundary map $B$ that defines cyclic homology (cf. Section 1.4 below). Our goal is to compare cyclic and mixed homology, and we will do so for more general deformations of $d$ by polynomials in $\xi$ :

Definition 1.5. Given any mixed complex $(\Omega, b, d)$ and a sequence of polynomials $c_{n} \in k[x]$, we define

$$
B_{n}:=c_{n} d_{n}, \quad v_{n}:=b_{n+1} B_{n}+B_{n-1} b_{n} .
$$


Our main result is the following:

Theorem 1.6. If all $c_{n} \in k[x]$ are invertible in $k \llbracket x \rrbracket$ and $(\Omega, b, B)$ is a homological skyscraper, then for all $n \geqslant 0$, there are canonical short exact sequences

$$
\begin{gathered}
0 \rightarrow \operatorname{ker} \pi_{n} \rightarrow \mathrm{H}_{n}(\Omega, b, d) \rightarrow \mathrm{H}_{n}(\hat{\Omega}, \hat{b}, \hat{B}) / \operatorname{ker} \pi_{n} \rightarrow 0, \\
0 \rightarrow \mathrm{H}_{n}(\Omega, b, B) \rightarrow \mathrm{H}_{n}(\hat{\Omega}, \hat{b}, \hat{B}) \rightarrow \mathrm{H}_{n-1}(\operatorname{im} \xi, b, B) \rightarrow 0,
\end{gathered}
$$

where $\pi_{n}$ is the canonical map $\mathrm{H}_{n}(\hat{\Omega}, \hat{b}, \hat{B}) \rightarrow \mathrm{H}_{n}(\hat{\Omega} / \operatorname{im} \hat{b}, 0, \hat{B})$.

The maps in (2) are induced by the embedding $\operatorname{im} \xi \rightarrow \Omega$ and the quotient $\Omega \rightarrow \hat{\Omega}$; those in (1) will be described in Section 2.6.

Thus if the two short exact sequences split, then choosing a split for both yields an isomorphism

$$
\mathrm{H}_{n}(\Omega, b, d) \cong \mathrm{H}_{n}(\Omega, b, B) \oplus \mathrm{H}_{n-1}(\operatorname{im} \xi, b, B) .
$$

Examples 2.18, 2.19, respectively 2.20 at the end of the paper illustrate the nontriviality of Theorem 1.6 by exhibiting mixed complexes for which $\mathrm{H}(\operatorname{im} \xi, b, B) \neq 0$ respectively $\operatorname{ker} \pi \neq 0$.

A key step in the proof is the following computation that relates the two spectral parameters $\xi$ and $v$; as we will explain below, this extends a result of Cuntz and Quillen.

Proposition 1.7. We have

$$
v_{n}=\xi_{n} c_{n}-d_{n-1} b_{n} f_{n}=b_{n+1} d_{n} f_{n}+\xi_{n} c_{n-1},
$$

where $f_{n}:=c_{n}-c_{n-1}$, and

$$
\left(v_{n}-\xi_{n} c_{n}\right)\left(v_{n}-\xi_{n} c_{n-1}\right)=0
$$

\subsection{Cyclic homology}

The most important choice for the polynomials $c_{n}$ leads to the definition of cyclic homology:

Definition 1.8. If

$$
c_{n}=\sum_{i=0}^{n}(1-x)^{i}=\frac{1-(1-x)^{n+1}}{x}=\sum_{i=0}^{n}(-1)^{i}\left(\begin{array}{c}
n+1 \\
i+1
\end{array}\right) x^{i},
$$

then $B_{n}=d_{n} \sum_{i=0}^{n}\left(\mathrm{id}-\xi_{n}\right)^{i}$ is called the Connes coboundary map and $\mathrm{H}(\Omega, b, B)$ the cyclic homology of $\Omega$; furthermore, $\Omega$ is said to be a cyclic complex if $v=b B+B b=0$.

Theorem 1.6 relates, in particular, the mixed homology of a cyclic complex to its cyclic homology, as long as the constant coefficients $n+1$ of $c_{n}$ are invertible in the ground ring $k$. If $v=0$, we have that $\mathrm{H}_{n}(\operatorname{im} \xi, b, B)=\bigoplus_{i \geqslant 0} \operatorname{ker} \xi_{n-2 i} \cap \operatorname{im} \xi_{n-2 i}$.

Corollary 1.9. If $(\Omega, b, d)$ is a cyclic complex of $\mathbb{Q}$-vector spaces, then there are (noncanonical) isomorphisms of vector spaces

$$
\mathrm{H}_{n}(\Omega, b, d) \cong \mathrm{H}_{n}(\Omega, b, B) \oplus \bigoplus_{i \geqslant 0} \operatorname{ker} \xi_{n-1-2 i} \cap \operatorname{im} \xi_{n-1-2 i} .
$$


This applies, in particular, to the noncommutative differential forms over a unital associative algebra $A$. Here $(\Omega, b)$ is the normalised Hochschild chain complex of $A$, that is,

$$
\Omega_{n}:=A \otimes_{k}(A / k)^{\otimes_{k} n}
$$

where $k$ is embedded into $A$ as scalar multiples of the unit element, and $b_{n}$ is induced by the map

$$
\begin{aligned}
a_{0} \otimes_{k} a_{1} \otimes_{k} \cdots \otimes_{k} a_{n} \mapsto & a_{0} a_{1} \otimes_{k} a_{2} \otimes_{k} \cdots \otimes_{k} a_{n} \\
& -a_{0} \otimes_{k} a_{1} a_{2} \otimes_{k} \cdots \otimes_{k} a_{n}+\cdots \\
& +(-1)^{n-1} a_{0} \otimes_{k} a_{1} \otimes_{k} \cdots \otimes_{k} a_{n-1} a_{n} \\
& +(-1)^{n} a_{n} a_{0} \otimes_{k} a_{1} \otimes_{k} \cdots \otimes_{k} a_{n-1} .
\end{aligned}
$$

The coboundary map is the noncommutative De Rham differential $d_{n}$ which is induced by

$$
d_{n}\left(a_{0} \otimes_{k} \cdots \otimes_{k} a_{n}\right):=1 \otimes_{k} a_{0} \otimes_{k} \cdots \otimes_{k} a_{n}
$$

This is a cyclic complex, and $\mathrm{H}(\Omega, b, B)$ is the cyclic homology $H C(A)$ of the algebra $A$ $[3,15]$.

Considering again a general mixed complex $(\Omega, b, d)$, the formulas from Proposition 1.7 reduce with $c_{n}$ as in Definition 1.8 to

$$
\begin{gathered}
T_{n}=\left(\mathrm{id}-b_{n+1} d_{n}\right) \kappa_{n}^{n}, \quad \kappa_{n}^{n+1}=T_{n}\left(\mathrm{id}-d_{n-1} b_{n}\right), \\
\left(T_{n}-\kappa_{n}^{n+1}\right)\left(T_{n}-\kappa_{n}^{n}\right)=0
\end{gathered}
$$

where

$$
\kappa_{n}:=\mathrm{id}-\xi_{n}, \quad T_{n}:=\mathrm{id}-v_{n}
$$

are the Karoubi operators of the two mixed complexes $(\Omega, b, d)$ and $(\Omega, b, B)$ respectively. This generalises [6, Proposition 3.1] to arbitrary mixed complexes and, in particular, to all cyclic ones, where $T=\mathrm{id}$ (Cuntz and Quillen only considered the example of noncommutative differential forms).

Our original motivation for the present work was to extend the results of Cuntz and Quillen and related work to the so-called twisted cyclic homology introduced by Kustermans, Murphy and Tuset [14], see Example 2.8 for details. This and the Hopfcyclic homology discovered by Connes-Moscovici [4] and Crainic [5] can be viewed as a special case of Hopf-cyclic homology with coefficients in anti Yetter-Drinfeld modules, as introduced by Hajac, Khalkhali, Rangipour and Sommerhäuser [10]. Early versions and special instances of the main results of our paper were key tools in the computation of the twisted cyclic homology of quantum $S L(2)$ due to Hadfield and the first author [9]. Another source of motivation was the work of Shapiro [16] who investigated the approach via noncommutative differential forms. Although the results as formulated here are fairly technical, we felt it worthwhile to present them in full generality from the viewpoint of mixed complexes and hope they will find new applications in other settings in the future. 


\section{Proofs and further material}

\subsection{Quasiisomorphisms}

Before beginning the proofs of the main results, we remark that what one should call a quasiisomorphism (or weak equivalence) of mixed complexes is a subtle question that depends on one's aims (see, e.g., [15, Section 2.5.14] and [8] for two different choices). We will, however, only encounter the simple case covered by the following proposition, which is a straightforward generalisation of [15, Corollary 2.2.3]:

Proposition 2.1. A morphism $\varphi:(\Omega, b, d) \rightarrow\left(\Omega^{\prime}, b^{\prime}, d^{\prime}\right)$ of mixed complexes, with $b d+d b=b^{\prime} d^{\prime}+d^{\prime} b^{\prime}=0$, induces an isomorphism on homology if and only if it induces an isomorphism on mixed homology.

Example 2.2. Observe that the analogue of the proposition for cohomological quasiisomorphisms fails: consider for example the two mixed complexes

$$
\Omega_{n}:=\left\{\begin{array}{ll}
\mathbb{C} & n=0, \\
0 & n>0,
\end{array} \quad \Omega_{m}^{\prime}:=\mathbb{C}, \quad m \in \mathbb{N},\right.
$$

with $b_{n}=d_{n}=b_{n}^{\prime}=0$ and

$$
d_{n}^{\prime}:=\left\{\begin{array}{ll}
0 & n=2 k, \\
\text { id } & n=2 k+1,
\end{array} \quad k \in \mathbb{N} .\right.
$$

We have

$$
\mathrm{H}^{n}(\Omega, 0)=\Omega_{n} \cong \mathrm{H}^{n}\left(\Omega^{\prime}, d^{\prime}\right)
$$

so the map

$$
\varphi_{n}:= \begin{cases}\mathrm{id} & n=0 \\ 0 & n>0\end{cases}
$$

is a quasiisomorphism of cochain complexes:

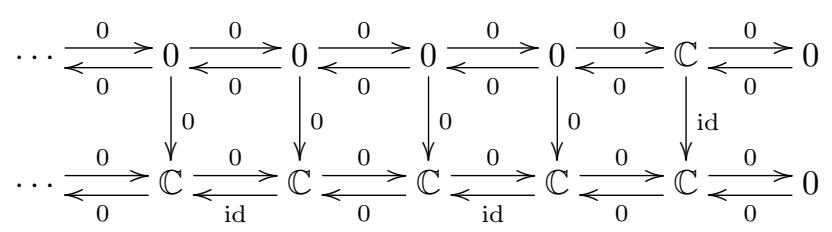

However, one obtains by direct inspection

$$
\mathrm{H}_{n}\left(\Omega^{\prime}, 0, b^{\prime}\right) \cong \mathbb{C}, \quad \mathrm{H}_{n}(\Omega, 0,0) \cong\left\{\begin{array}{ll}
\mathbb{C} & n=2 k, \\
0 & n=2 k+1,
\end{array} \quad k \in \mathbb{N}\right.
$$

Remark 2.3. The moral is that, although the rôles of $d$ and $b$ are entirely symmetric in $\Omega$, this symmetry is broken in the definition of mixed homology, as the action of $d$ is somewhat artificially cut off on $\hat{\Omega}_{n} \subset \mathrm{T}_{n}(\Omega)$. This changes when one considers the $\mathbb{Z}_{2}$-graded periodic homology theories; however, then there are two variants:

$$
\mathrm{T}_{s}^{\text {per }, \Pi}(\Omega):=\prod_{j \in \mathbb{N}} \hat{\Omega}_{s+2 j}, \quad \mathrm{~T}_{s}^{\mathrm{per}, \oplus}(\Omega):=\bigoplus_{j \in \mathbb{N}} \hat{\Omega}_{s+2 j}, \quad s \in \mathbb{Z}_{2} .
$$

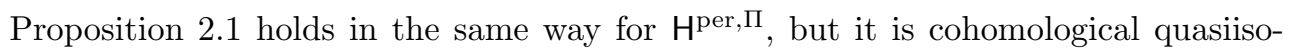
morphisms rather than homological ones which induce isomorphisms in $\mathrm{H}^{\mathrm{per}, \oplus}$. 


\subsection{The proof of Proposition 1.7}

We now develop the theory that will lead to a proof of Theorem 1.6. The steps are illustrated using the example of cyclic homology, and the first one is the proof of Proposition 1.7 in which we relate the maps $\xi$ and $v$ :

Proof of Proposition 1.7. The first equation is obtained by straightforward computation:

$$
\begin{aligned}
v_{n} & =b_{n+1} B_{n}+B_{n-1} b_{n}=b_{n+1} c_{n} d_{n}+c_{n-1} d_{n-1} b_{n} \\
& =b_{n+1} d_{n} c_{n}+d_{n-1} b_{n} c_{n-1}=\left(\xi_{n}-d_{n-1} b_{n}\right) c_{n}+d_{n-1} b_{n} c_{n-1} \\
& =b_{n+1} d_{n} c_{n}+\left(\xi_{n}-b_{n+1} d_{n}\right) c_{n-1} .
\end{aligned}
$$

Thus the first factor in (4) equals $-d_{n-1} b_{n} f_{n+1}$ and the second one $b_{n+1} d_{n} f_{n}$, so their product equals 0 as $b_{n} b_{n+1}=0$.

Remark 2.4. If one perturbs not just $d_{n}$ to $B_{n}=c_{n} d_{n}$ but also $b_{n}$ to $D_{n}:=a_{n} b_{n}$ for some polynomials $a_{n} \in k[x]$, then one has

$$
B_{n-1} D_{n}+D_{n+1} B_{n}=\xi_{n} a_{n+1} c_{n}-d_{n-1} b_{n} f_{n}=b_{n+1} d_{n} f_{n}+\xi_{n} a_{n} c_{n-1},
$$

with $f_{n}=a_{n+1} c_{n}-a_{n} c_{n-1}$. That is, one obtains $v$ but for $d$ perturbed by the polynomials $a_{n+1} c_{n}$ and in this sense it is sufficient to focus on deformations of $d$ alone.

Example 2.5. In the case of cyclic homology (cf. Section 1.4), we obtain

$$
c_{n}=\frac{1-y^{n+1}}{1-y}, \quad f_{n}=y^{n}, \quad y:=1-x .
$$

Inserting this into the formulas in Proposition 1.7 yields the formulas (7)-(9) from Section 1.4.

\subsection{The quasiisomorphism $\Omega \rightarrow \bar{\Omega}$}

As part of the assumptions of Theorem 1.6, $(\Omega, b, B)$ is a homological skyscraper, so $(\operatorname{im} v, b)$ has trivial homology. We now use this fact to relate the mixed homology of $\Omega$ to that of the quotients

$$
\bar{\Omega}:=\Omega / \operatorname{im} v, \quad \tilde{\Omega}:=\Omega /(\operatorname{im} \xi+\operatorname{im} v) .
$$

In the sequel, $\bar{d}, \bar{b}, \bar{\xi}$ and $\tilde{b}, \tilde{d}, \tilde{\xi}$ refer to the structure maps on $\bar{\Omega}$, respectively $\tilde{\Omega}$.

Lemma 2.6. ( $\operatorname{im} \xi \cap \operatorname{im} v, b)$ has trivial homology.

Proof. If $x \in(\operatorname{im} v \cap \operatorname{im} \xi)_{n}$ and $b_{n} x=0$, then as (im $\left.v, b\right)$ has no homology, there is $y \in \Omega_{n+1}$ with $x=b_{n+1} v_{n+1} y$. By Proposition 1.7 , this equals $b_{n+1} \xi_{n+1} c_{n} y$, so $x \in b(\operatorname{im} v \cap \operatorname{im} \xi)_{n+1}$.

Lemma 2.7. The canonical quotient $\hat{\Omega} \rightarrow \tilde{\Omega}$ is a quasiisomorphism of chain complexes. In particular, the quotient homomorphism $(\Omega, b, d) \rightarrow(\bar{\Omega}, \bar{b}, \bar{d})$ induces isomorphisms $\mathrm{H}(\Omega, b, d)=\mathrm{H}(\hat{\Omega}, \hat{b}, \hat{d}) \cong \mathrm{H}(\bar{\Omega}, \bar{b}, \bar{d})=\mathrm{H}(\tilde{\Omega}, \tilde{b}, \tilde{d})$.

Proof. We need to show that the kernel

$$
\operatorname{im} v /(\operatorname{im} \xi \cap \operatorname{im} v)
$$

has trivial homology. However, this follows from the fact that $\operatorname{im} v$ and $\operatorname{im} \xi \cap \operatorname{im} v$ have trivial homology (im $v$ has trivial homology by the assumption that $(\Omega, b, B$ ) is 
a homological skyscraper, and $\operatorname{im} \xi \cap \operatorname{im} v$ has trivial homology by Lemma 2.6). The second claim now follows from Proposition 2.1.

Example 2.8. If $\Omega$ is a cyclic complex and $\mathrm{H}(\Omega, b, B)$ is its cyclic homology as in Definition 1.8 , then $v=0$ and $\Omega=\bar{\Omega}$, so the above lemma becomes trivial. However, let $A$ be an associative algebra and ${ }_{\sigma} A$ be the $A$-bimodule which is $A$ as a right $A$-module but whose left action is given by $x \triangleright y:=\sigma(x) y$ for some algebra endomorphism $\sigma$. Now consider the noncommutative differential forms over $A$ as defined in (5), but with the boundary map $b$ that computes the Hochschild homology of $A$ with coefficients in ${ }_{\sigma} A$. Explicitly, $b$ is given by

$$
\begin{aligned}
a_{0} \otimes_{k} a_{1} \otimes_{k} \cdots \otimes_{k} a_{n} \mapsto & a_{0} a_{1} \otimes_{k} a_{2} \otimes_{k} \cdots \otimes_{k} a_{n} \\
& -a_{0} \otimes_{k} a_{1} a_{2} \otimes_{k} \cdots \otimes_{k} a_{n}+\cdots \\
& +(-1)^{n-1} a_{0} \otimes_{k} a_{1} \otimes_{k} \cdots \otimes_{k} a_{n-1} a_{n} \\
& +(-1)^{n} \sigma\left(a_{n}\right) a_{0} \otimes_{k} a_{1} \otimes_{k} \cdots \otimes_{k} a_{n-1} .
\end{aligned}
$$

With $d_{n}$ from (6) and $c_{n}$ as in Definition 1.8 , the operator $B$ is induced by

$$
B_{n}\left(a_{0} \otimes_{k} \cdots \otimes_{k} a_{n}\right)=\sum_{i=0}^{n} 1 \otimes_{k} t^{i}\left(a_{0} \otimes_{k} \cdots \otimes_{k} a_{n}\right),
$$

where

$$
t\left(a_{0} \otimes_{k} \cdots \otimes_{k} a_{n}\right):=(-1)^{n} \sigma\left(a_{n}\right) \otimes_{k} a_{0} \otimes_{k} \cdots \otimes_{k} a_{n-1} .
$$

In this case, $\mathrm{H}(\Omega, b, B)$ is the twisted cyclic homology $H C^{\sigma}(A)$ of $A$ that was first considered by Kustermans, Murphy and Tuset [14]. The operators $\xi$ and $v$ are given in this case by

$$
\begin{aligned}
\xi\left(a_{0} \otimes_{k} \cdots \otimes_{k} a_{n}\right)= & a_{0} \otimes_{k} \cdots \otimes_{k} a_{n}-t\left(a_{0} \otimes_{k} \cdots \otimes_{k} a_{n}\right) \\
& +(-1)^{n} 1 \otimes_{k} \sigma\left(a_{n}\right) a_{0} \otimes_{k} \cdots \otimes_{k} a_{n-1}, \\
v\left(a_{0} \otimes_{k} \cdots \otimes_{k} a_{n}\right)= & a_{0} \otimes_{k} \cdots \otimes_{k} a_{n}-\sigma\left(a_{0}\right) \otimes_{k} \cdots \otimes_{k} \sigma\left(a_{n}\right) .
\end{aligned}
$$

In particular, $\Omega$ is cyclic if and only if $\sigma=\mathrm{id}$.

To generalise the theory of Cuntz and Quillen (which deals only with the case where $\sigma=\mathrm{id}$ ) to this setting was one of our original aims, motivated, in particular, by Shapiro's extension [16] of Karoubi's noncommutative De Rham theory.

\subsection{The quasiisomorphism $\operatorname{ker} \bar{\xi}^{2} \rightarrow \bar{\Omega}$}

From now on, we will study the mixed complex $\bar{\Omega}$ in further detail. This is where the second main assumption of Theorem 1.6 becomes relevant, namely that the constant coefficients of the polynomials $c_{n}$ are all invertible. We tacitly assume this for the rest of the paper.

Lemma 2.9. We have

$$
\bar{\Omega}=\operatorname{ker} \bar{\xi}^{2} \oplus \operatorname{im} \bar{\xi}^{2} .
$$

Proof. Assuming that all $c_{n}$ are invertible in $k \llbracket x \rrbracket$ means their constant coefficients are invertible in $k$. Hence also $c_{n-1} c_{n}$ has an invertible constant coefficient $\varepsilon_{n} \in k$. 
Let $\delta_{n}, \gamma_{n} \in k$ be its linear and quadratic coefficient,

$$
c_{n-1} c_{n}=\varepsilon_{n}+\delta_{n} x+\gamma_{n} x^{2}+\cdots
$$

and define

$$
\bar{p}_{n}:=\varepsilon_{n}^{-2}\left(\varepsilon_{n}-\delta_{n} \bar{\xi}\right) \bar{c}_{n-1} \bar{c}_{n}=1+\left(\frac{\gamma_{n}}{\varepsilon_{n}}-\frac{\delta_{n}^{2}}{\varepsilon_{n}^{2}}\right) \bar{\xi}^{2}+\cdots,
$$

where $\bar{c}_{n}: \bar{\Omega}_{n} \rightarrow \bar{\Omega}_{n}$ is the map obtained by inserting $\bar{\xi}$ into $c_{n}$.

Since $v$ induces the trivial map on $\bar{\Omega}=\Omega /$ im $v$, Proposition 1.7 implies

$$
\bar{\xi}^{2} \bar{c}_{n-1} \bar{c}_{n}=\bar{c}_{n-1} \bar{c}_{n} \bar{\xi}^{2}=0,
$$

so we get

$$
\operatorname{im} \bar{p}_{n} \subset \operatorname{im} \bar{c}_{n-1} \bar{c}_{n} \subset \operatorname{ker} \bar{\xi}^{2}, \quad \operatorname{im} \bar{\xi}^{2} \subset \operatorname{ker} \bar{c}_{n-1} \bar{c}_{n} \subset \operatorname{ker} \bar{p}_{n} .
$$

Conversely, $\bar{p}_{n}$ acts, by definition, as the identity on $\operatorname{ker} \bar{\xi}^{2}$, so we also have that $\operatorname{ker} \bar{\xi}^{2} \subset \operatorname{im} \bar{p}_{n}$, and on $\operatorname{ker} \bar{p}_{n}$ we have $1=\bar{\xi}^{2}\left(\frac{\delta_{n}^{2}}{\varepsilon_{n}^{2}}-\frac{\gamma_{n}}{\varepsilon_{n}}\right)+\cdots$, so $\operatorname{ker} \bar{p}_{n} \subset \operatorname{im} \bar{\xi}^{2}$. It follows that $\operatorname{ker} \bar{\xi}^{2}=\operatorname{im} \bar{p}_{n}$ and $\operatorname{im} \bar{\xi}^{2}=\operatorname{ker} \bar{p}_{n}$, and also that $\bar{p}_{n}^{2}=\bar{p}_{n}$, so that we have $\Omega / \operatorname{im} v=\operatorname{im} \bar{p}_{n} \oplus \operatorname{ker} \bar{p}_{n}$.

Lemma 2.10. The inclusion $\operatorname{ker} \bar{\xi}^{2} \rightarrow \bar{\Omega}$ induces isomorphisms

$$
\mathrm{H}(\bar{\Omega}, \bar{b}, \bar{d}) \cong \mathrm{H}\left(\operatorname{ker} \bar{\xi}^{2}, \bar{b}, \bar{d}\right), \quad \mathrm{H}(\bar{\Omega}, \bar{b}, \bar{B}) \cong \mathrm{H}\left(\operatorname{ker} \bar{\xi}^{2}, \bar{b}, \bar{B}\right) .
$$

Proof. As $\bar{\xi}$ is a morphism of mixed complexes, (10) is a decomposition of mixed complexes. Since we have

$$
\operatorname{ker} \bar{\xi} \subset \operatorname{ker} \bar{\xi}^{2}, \quad \operatorname{im} \bar{\xi}^{2} \subset \operatorname{im} \bar{\xi},
$$

we conclude, using Lemma 2.9 in the last step, that

$$
\tilde{\Omega}=\Omega /(\operatorname{im} v+\operatorname{im} \xi) \cong \bar{\Omega} / \operatorname{im} \bar{\xi} \cong \operatorname{ker} \bar{\xi}^{2} / \operatorname{im} \bar{\xi},
$$

so the first isomorphism is obvious. Equation (10) also implies that $\bar{\xi}^{2}$ and hence $\bar{\xi}$ is invertible on $\operatorname{im} \bar{\xi}^{2}$, so $\operatorname{im} \bar{\xi}^{2}$ is contractible as explained in Section 1.2. This means the inclusion is a quasiisomorphism with respect to $\bar{b}$. Hence the second isomorphism follows from Proposition 2.1.

For later use, we record here another elementary consequence of Lemma 2.9:

Corollary 2.11. We have $\operatorname{im} \bar{\xi} \cap \operatorname{ker} \bar{\xi}^{2}=\operatorname{im} \bar{\xi} \cap \operatorname{ker} \bar{\xi}$.

Proof. Given $y=\bar{\xi}(x) \in \operatorname{ker} \bar{\xi}^{2}$, write $x$ as $x=v+w$ with $v \in \operatorname{ker} \bar{\xi}^{2}$ and $w \in \operatorname{im} \bar{\xi}^{2}$. Then $\bar{\xi}^{2}(y)=0$ means $\bar{\xi}^{3}(v)+\bar{\xi}^{3}(w)=0$; so $\bar{\xi}^{2}(v)=0$ yields $\bar{\xi}^{3}(w)=0$. However, $\bar{\xi}$ is injective on $\operatorname{im} \xi^{2}$ as already remarked in the previous proof, so $w=0$, hence $x=v$, so $\bar{\xi}(y)=\bar{\xi}^{2}(x)=\bar{\xi}^{2}(v)=0$.

Remark 2.12. All the above computations are abstractions of those made by Cuntz and Quillen for the noncommutative differential forms over an associative algebra [6]. Informally speaking, the message of Lemma 2.10 can be stated as follows: the "best" mixed complexes are those where $\xi=0$, as one can compute $\mathrm{H}(\Omega, b, d)$ straight from $\Omega$ using a spectral sequence. The second best case is $\Omega=\operatorname{ker} \xi \oplus \operatorname{im} \xi$; as mentioned after Definition 1.2 this means $\xi$ vanishes in a strong homotopical sense. Lemma 2.10 tells us that in general $\xi^{2}$ vanishes in this homotopical sense, so $\xi$ is homotopically infinitesimal. 


\subsection{The second exact sequence}

We now will derive the second of the two short exact sequences in Theorem 1.6.

First, we need the following computation:

Lemma 2.13. On $\operatorname{ker} \bar{\xi}^{2}$, we have $\bar{b} \bar{\xi}=\bar{d} \bar{\xi}=0, \bar{B}_{n}:=\bar{c}_{n} \bar{d}_{n}=\beta_{n} \bar{d}_{n}$ for all $n \geqslant 0$, where $\beta_{n} \in k$ is the constant coefficient of $c_{n}$, and we have

$$
\bar{\xi}_{n}=\left(1-\frac{\beta_{n-1}}{\beta_{n}}\right) \bar{d}_{n-1} \bar{b}_{n}=\left(1-\frac{\beta_{n}}{\beta_{n-1}}\right) \bar{b}_{n+1} \bar{d}_{n} .
$$

Proof. Multiplying the second expression for $v=0$ in (3) in Proposition 1.7 on the left by $b_{n}$ and using $\bar{\xi}^{2}=0$ gives

$$
\bar{b}_{n} \bar{\xi} \bar{c}_{n-1}=\beta_{n-1} \bar{b}_{n} \bar{\xi}=0,
$$

so $\bar{b} \bar{\xi}=0$ as all $\beta_{n}$ are invertible. Similarly, one obtains $\bar{d} \bar{\xi}=0$. That $\bar{B}_{n}=\beta_{n} d_{n}$ is an immediate consequence, and the formulas for $\bar{\xi}_{n}$ are obtained by direct computation:

$$
\begin{aligned}
\bar{\xi}_{n} & =\bar{d}_{n-1} \bar{b}_{n}+\bar{b}_{n+1} \bar{d}_{n}=\bar{d}_{n-1} \bar{b}_{n}+\beta_{n}^{-1} \bar{b}_{n+1} \bar{B}_{n} \\
& =\bar{d}_{n-1} \bar{b}_{n}-\beta_{n}^{-1} \bar{B}_{n-1} \bar{b}_{n}=\left(1-\frac{\beta_{n-1}}{\beta_{n}}\right) \bar{d}_{n-1} \bar{b}_{n}
\end{aligned}
$$

and similarly $\bar{\xi}_{n}=\left(1-\frac{\beta_{n}}{\beta_{n-1}}\right) \bar{b}_{n+1} \bar{d}_{n}$.

Additionally, we will utilise the following general statement (recall $\hat{\Omega}=\Omega / \operatorname{im} \xi$ ):

Lemma 2.14. If $(\Omega, b, d)$ is a mixed complex with $\xi^{2}=v=0$, then for all $n \geqslant 0$, there are short exact sequences

$$
0 \longrightarrow \mathrm{H}_{n}(\Omega, b, B) \longrightarrow \mathrm{H}_{n}(\hat{\Omega}, \hat{b}, \hat{B}) \longrightarrow \bigoplus_{i \geqslant 0} \operatorname{im} \xi_{n-1-2 i} \longrightarrow 0
$$

Proof. The short exact sequence

$$
0 \longrightarrow(\operatorname{im} \xi, b, B) \longrightarrow(\Omega, b, B) \longrightarrow(\hat{\Omega}, \hat{b}, \hat{B}) \longrightarrow 0
$$

of mixed complexes induces short exact sequences of the total complexes

$$
0 \longrightarrow \mathrm{T}(\mathrm{im} \xi) \longrightarrow \mathrm{T}(\Omega) \longrightarrow \mathrm{T}(\hat{\Omega}) \longrightarrow 0,
$$

whose differential is $b+B$ (recall that $v=b B+B b=0$ so that $\mathrm{T}_{n}(\Omega)=\Omega_{n} \oplus$ $\Omega_{n-2} \oplus \cdots$ here). However, by Lemma $2.13, b+B$ vanishes on $\operatorname{im} \xi$, so that $\mathrm{T}(\operatorname{im} \xi)$ is its own homology. Furthermore, the inclusion $\operatorname{im} \xi \rightarrow \Omega$ induces the trivial map on homology, as Lemma 2.13 implies

$$
\left(\xi_{n} x_{n}, \xi_{n-2} x_{n-2}, \ldots\right)=(b+B)\left(\left(1-\frac{\beta_{n}}{\beta_{n-1}}\right) d_{n} x_{n},\left(1-\frac{\beta_{n-2}}{\beta_{n-3}}\right) d_{n-2} x_{n-2}, \ldots\right),
$$

so indeed, the homology class of an element in $\mathrm{T}(\mathrm{im} \xi)$ becomes trivial in $\mathrm{H}(\Omega, b, B)$. Therefore, the long exact homology sequence induced by (12) splits up into the short exact sequences stated in the lemma. 
Proof of Theorem 1.6 (2). We apply Lemma 2.14 to $\operatorname{ker} \bar{\xi}^{2} \subset \bar{\Omega}$. This yields short exact sequences

$$
0 \longrightarrow \mathrm{H}_{n}\left(\operatorname{ker} \bar{\xi}^{2}, \bar{b}, \bar{B}\right) \longrightarrow \mathrm{H}_{n}\left(\operatorname{ker} \bar{\xi}^{2} / \mathrm{I}, \bar{b}, \bar{B}\right) \longrightarrow \bigoplus_{i \geqslant 0} \mathrm{I}_{n-1-2 i} \longrightarrow 0,
$$

where we abbreviate

$$
\mathrm{I}:=\operatorname{im} \bar{\xi} \cap \operatorname{ker} \bar{\xi}^{2}=\operatorname{im} \bar{\xi} \cap \operatorname{ker} \bar{\xi},
$$

the second equality having been proved in Corollary 2.9. Note that, by abuse of notation, we did not introduce yet a new notation for the maps induced by $\bar{b}, \bar{B}$ on the quotient by I.

In view of (11), we have a commutative diagram

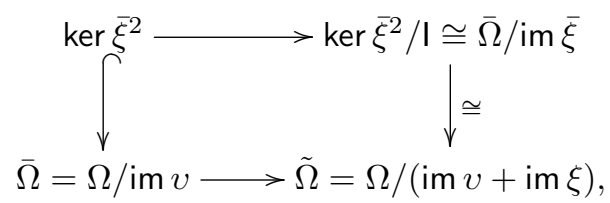

where the horizontal maps are the canonical projections, the left vertical map is the inclusion, and the right vertical map is an isomorphism induced by this inclusion.

By Lemma 2.10, the left vertical arrow induces an isomorphism

$$
\mathrm{H}\left(\operatorname{ker} \bar{\xi}^{2}, \bar{b}, \bar{B}\right) \cong \mathrm{H}(\bar{\Omega}, \bar{b}, \bar{B})=\mathrm{H}(\Omega, b, B) .
$$

Similarly, the right vertical isomorphism yields an isomorphism

$$
\mathrm{H}\left(\operatorname{ker} \bar{\xi}^{2} / \mathrm{I}, \bar{b}, \bar{B}\right) \cong \mathrm{H}(\tilde{\Omega}, \tilde{b}, \tilde{B})=\mathrm{H}(\hat{\Omega}, \hat{b}, \hat{B}) .
$$

These isomorphisms are compatible with the horizontal quotient maps in the diagram. In other words, the injectivity of the embedding $\mathrm{H}\left(\operatorname{ker} \bar{\xi}^{2}, \bar{b}, \bar{B}\right) \rightarrow \mathrm{H}\left(\operatorname{ker} \bar{\xi}^{2} / \mathrm{I}, \bar{b}, \bar{B}\right)$ established in (13) transfers to injectivity of the map $\mathrm{H}(\Omega, b, B) \rightarrow \mathrm{H}(\hat{\Omega}, \hat{b}, \hat{B})$ induced by the quotient $\Omega \rightarrow \hat{\Omega}$.

Now, since the canonical map $\mathrm{H}(\Omega, b, B) \rightarrow \mathrm{H}(\hat{\Omega}, \hat{b}, \hat{B})$ is injective, the long exact homology sequence resulting from the short exact sequence

$$
0 \rightarrow \operatorname{im} \xi \rightarrow \Omega \rightarrow \hat{\Omega} \rightarrow 0
$$

splits into the short exact sequences stated in the theorem.

Example 2.15. When considering the cyclic homology of a cyclic complex, we have $v=0$, hence $\bar{\xi}=\xi$ and we obtain

$$
\mathrm{H}_{n}(\operatorname{im} \xi, b, B)=\bigoplus_{i \geqslant 0} \mathrm{I}_{n-2 i}=\bigoplus_{i \geqslant 0} \operatorname{ker} \xi_{n-2 i} \cap \operatorname{im} \xi_{n-2 i} .
$$

\subsection{The first exact sequence}

Recall that when $(\Omega, b, B)$ is a homological skyscraper, we have $\mathbf{H}(\Omega, b, d) \cong$ $\mathrm{H}(\tilde{\Omega}, \tilde{b}, \tilde{d})$ by Lemma 2.7 , hence without loss of generality, we work with $\tilde{\Omega}$ instead of $\Omega$ from now on.

The bulk of the remaining computations needed to prove Theorem 1.6 are performed in the following lemma: 
Lemma 2.16. The map $\varphi_{n}: \mathrm{T}(\tilde{\Omega}) \rightarrow \mathrm{T}(\tilde{\Omega})$ given by

$$
\left(x_{n}, x_{n-2}, \ldots\right) \mapsto\left(u_{n}, u_{n-2}, \ldots\right):=\left(x_{n}, \beta_{n-2}^{-1} x_{n-2}, \beta_{n-2}^{-1} \beta_{n-4}^{-1} x_{n-4}, \ldots\right)
$$

induces isomorphisms

$$
\begin{aligned}
& \mathrm{H}(\tilde{\Omega} / \operatorname{im} \tilde{b}, 0, \tilde{d}) \cong \mathrm{H}(\tilde{\Omega} / \operatorname{im} \tilde{b}, 0, \tilde{B}), \quad \mathrm{H}(\operatorname{im} \tilde{b}, 0, \tilde{d}) \cong \mathrm{H}(\operatorname{im} \tilde{b}, 0, \tilde{B}), \\
& \operatorname{im}(\mathrm{H}(\tilde{\Omega}, \tilde{b}, \tilde{d}) \rightarrow \mathrm{H}(\tilde{\Omega} / \operatorname{im} \tilde{b}, 0, \tilde{d})) \cong \operatorname{im}(\mathrm{H}(\tilde{\Omega}, \tilde{b}, \tilde{B}) \rightarrow \mathrm{H}(\tilde{\Omega} / \operatorname{im} \tilde{b}, 0, \tilde{B})), \\
& \operatorname{ker}(\mathrm{H}(\tilde{\Omega}, \tilde{b}, \tilde{d}) \rightarrow \mathrm{H}(\tilde{\Omega} / \operatorname{im} \tilde{b}, 0, \tilde{d})) \cong \operatorname{ker}(\mathrm{H}(\tilde{\Omega}, \tilde{b}, \tilde{B}) \rightarrow \mathrm{H}(\tilde{\Omega} / \operatorname{im} \tilde{b}, 0, \tilde{B})) .
\end{aligned}
$$

Proof. Explicitly, a class in $\mathrm{H}_{n}(\tilde{\Omega} / \operatorname{im} \tilde{b}, 0, \tilde{d})$ is represented by an element denoted $x=\left(x_{n}, x_{n-2}, \ldots\right) \in \mathrm{T}_{n}(\tilde{\Omega})$, such that there exists $y \in \mathrm{T}_{n}(\tilde{\Omega})$ with

$$
\tilde{b} x_{n}+\tilde{d} x_{n-2}=\tilde{b} y_{n}, \quad \tilde{b} x_{n-2}+\tilde{d} x_{n-4}=\tilde{b} y_{n-2}, \ldots
$$

The element $x$ represents the trivial homology class in $\mathrm{H}_{n}(\tilde{\Omega} / \mathrm{im} \tilde{b}, 0, \tilde{d})$ if and only if there are elements $z=\left(z_{n+1}, z_{n-1}, \ldots\right), t \in \mathrm{T}_{n+1}(\tilde{\Omega})$ such that

$$
\tilde{b} z_{n+1}+\tilde{d} z_{n-1}=x_{n}+\tilde{b} t_{n+1}, \quad \tilde{b} z_{n-1}+\tilde{d} z_{n-3}=x_{n-2}+\tilde{b} t_{n-1}, \ldots
$$

Recall that $\tilde{\xi}=0$ means that $\tilde{B}_{n}=\beta_{n} \tilde{d}_{n}$ where $\beta_{n} \in k$ is the constant coefficient of $c_{n}$. Hence $u=\varphi_{n}(x) \in \mathrm{T}_{n}(\tilde{\Omega})$ satisfies

$$
\tilde{b} u_{n}+\tilde{B} u_{n-2}=\tilde{b} v_{n}, \quad \tilde{b} u_{n-2}+\tilde{B} u_{n-4}=\tilde{b} v_{n-2}, \ldots
$$

where $v=\varphi_{n}(y)$.

Furthermore, (15) implies

$$
\tilde{b} w_{n+1}+\tilde{B} w_{n-1}=u_{n}+\tilde{b} s_{n+1}, \ldots
$$

with $w=\varphi_{n+1}(z), s=\varphi_{n+1}(t)$. This shows that $\varphi_{n}$ induces a well-defined map on homology, which is clearly bijective. Also, the image of $\mathrm{H}_{n}(\tilde{\Omega}, \tilde{b}, \tilde{d})$ in $\mathrm{H}_{n}(\tilde{\Omega} / \operatorname{im} \tilde{b}, 0, \tilde{d})$ consists of those classes that can be represented as above with $y=0$, and then $v=0$ means that the image in $\mathrm{H}_{n}(\tilde{\Omega} / \operatorname{im} \tilde{b}, 0, \tilde{B})$ is also in the image of $\mathrm{H}_{n}(\tilde{\Omega}, \tilde{b}, \tilde{d})$. The other isomorphisms follow in an exactly analogous way.

Remark 2.17. For most of the isomorphisms required in Lemma 2.16, there is little restriction on the particular isomorphism we use; we could, for example, take the identity instead of $\varphi$. However, this causes (14) to fail.

Proof of Theorem 1.6 (1). The short exact sequences of chain complexes

$$
0 \rightarrow \mathrm{T}(\operatorname{im} \tilde{b}) \rightarrow \mathrm{T}(\tilde{\Omega}) \rightarrow \mathrm{T}(\tilde{\Omega} / \operatorname{im} \tilde{b}) \rightarrow 0,
$$

with respect to $\tilde{b}+\tilde{d}$ and $\tilde{b}+\tilde{B}$ yield long exact sequences

$$
\cdots \rightarrow \mathrm{H}_{n+1}(\tilde{\Omega} / \operatorname{im} \tilde{b}, 0, \tilde{d}) \stackrel{\partial_{n+1}^{d}}{\longrightarrow} \mathrm{H}_{n}(\operatorname{im} \tilde{b}, 0, \tilde{d}) \rightarrow \mathrm{H}_{n}(\tilde{\Omega}, \tilde{b}, \tilde{d}) \rightarrow \cdots
$$

and

$$
\cdots \rightarrow \mathrm{H}_{n+1}(\tilde{\Omega} / \operatorname{im} \tilde{b}, 0, \tilde{B}) \stackrel{\partial_{n+1}^{B}}{\longrightarrow} \mathrm{H}_{n}(\operatorname{im} \tilde{b}, 0, \tilde{B}) \rightarrow \mathrm{H}_{n}(\tilde{\Omega}, \tilde{b}, \tilde{B}) \rightarrow \cdots
$$

These split into short exact sequences

$$
0 \rightarrow \mathrm{H}_{n}(\operatorname{im} \tilde{b}, 0, \tilde{d}) / \operatorname{im} \partial_{n+1}^{d} \rightarrow \mathrm{H}_{n}(\tilde{\Omega}, \tilde{b}, \tilde{d}) \rightarrow \operatorname{ker} \partial_{n}^{d} \rightarrow 0
$$


and

$$
0 \rightarrow \mathrm{H}_{n}(\operatorname{im} \tilde{b}, 0, \tilde{B}) / \operatorname{im} \partial_{n+1}^{B} \rightarrow \mathrm{H}_{n}(\tilde{\Omega}, \tilde{b}, \tilde{B}) \rightarrow \operatorname{ker} \partial_{n}^{B} \rightarrow 0 .
$$

If $\iota_{n}$ denotes the map $\mathrm{H}_{n}(\operatorname{im} \tilde{b}, 0, \tilde{B}) \rightarrow \mathrm{H}_{n}(\tilde{\Omega}, \tilde{b}, \tilde{B})$ induced by the inclusion and $\pi_{n}$ denotes the map $\mathrm{H}_{n}(\tilde{\Omega}, \tilde{b}, \tilde{B}) \rightarrow \mathrm{H}_{n}(\tilde{\Omega} / \mathrm{im} \tilde{b}, 0, \tilde{B})$, then by exactness we have

$$
\mathrm{H}_{n}(\operatorname{im} \tilde{b}, 0, \tilde{B}) / \operatorname{im} \partial_{n+1}^{B}=\mathrm{H}_{n}(\operatorname{im} \tilde{b}, 0, \tilde{B}) / \operatorname{ker} \iota_{n} \cong \operatorname{im} \iota_{n}=\operatorname{ker} \pi_{n}
$$

and

$$
\operatorname{ker} \partial_{n}^{B}=\operatorname{im} \pi_{n} \cong \mathrm{H}_{m}(\tilde{\Omega}, \tilde{b}, \tilde{B}) / \operatorname{ker} \pi_{n} .
$$

The theorem now follows in view of the isomorphisms (of $k$-modules)

$$
\mathrm{H}_{n}(\operatorname{im} \tilde{b}, 0, \tilde{d}) / \operatorname{im} \partial_{n+1}^{d} \cong \mathrm{H}_{n}(\operatorname{im} \tilde{b}, 0, \tilde{B}) / \operatorname{im} \partial_{n+1}^{B}, \quad \operatorname{ker} \partial_{n}^{d} \cong \operatorname{ker} \partial_{n}^{B}
$$

established in Lemma 2.16. Note that in the introduction we suppressed introducing $\tilde{\Omega}$, but by definition, we have $\mathrm{H}(\hat{\Omega}, \hat{b}, \hat{B})=\mathrm{H}(\tilde{\Omega}, \tilde{b}, \tilde{B})$.

Example 2.18. If $A$ is an exterior algebra in generators $x, y$ of degree 1 over a field $k$ of characteristic $\neq 2$, so that

$$
x^{2}=x y+y x=y^{2}=0,
$$

then the noncommutative differential forms $\Omega$ over $A$ are not a homological skyscraper. Indeed, the class of

$$
1 \otimes_{k} x y=\frac{1}{2} \xi\left(x \otimes_{k} y-y \otimes_{k} x\right)
$$

in $\mathrm{H}_{1}(\operatorname{im} \xi, b)$ is nontrivial - a straightforward computation shows that $\operatorname{im} b \xi$ has no component in $A_{0} \otimes_{k} A_{2}$, where $A_{d}$ is the degree $d$-component of $A$. As $\xi$ vanishes in degree $0,1 \otimes_{k} x y$ also defines a nontrivial class in $\mathrm{H}_{1}(\operatorname{im} \xi, b, d)=\mathrm{H}_{1}(\operatorname{im} \xi, b, B)$.

Example 2.19. For a nonstandard example of Theorem 1.6, let $k$ be any commutative ring, $q \in k$, and $R$ be the unital associative $k$-algebra generated by $x, y$ satisfying

$$
x^{2}=y^{2}=x y+q y x=0,
$$

so that $R$ is a free $k$-module with basis $\{1, x, y, y x\}$.

We obtain a mixed complex of $R$-modules with

$$
\Omega_{n}:= \begin{cases}R / R y x & n=0 \\ R & n>0\end{cases}
$$

and $b_{n}$ given by right multiplication by $x$ and $d_{n}$ given by right multiplication by $y$. With $c_{n}=q^{n}$, that is, $B_{n}$ given by right multiplication by $q^{n} y$, we obtain for $n>0$ and $r \in R=\Omega_{n}$

$$
\begin{aligned}
& \left(b_{n-1} B_{n}+B_{n-1} b_{n}\right) r=r\left(q^{n} y x+q^{n-1} x y\right)=0, \\
& \left(b_{n-1} d_{n}+d_{n-1} b_{n}\right) r=r(y x+x y)=(1-q) r y x,
\end{aligned}
$$

and for $n=0$

$$
\left(b_{1} B_{0}\right)(r+R y x)=\left(b_{1} d_{0}\right)(r+R y x)=r y x+R y x=0 .
$$

In particular,

$$
\mathrm{H}_{1}(\operatorname{im} \xi, b, B)=\operatorname{im} \xi_{1} \cong k / I,
$$


where $I \triangleleft k$ is the annihilator of $1-q$ in $k$.

We furthermore see

$$
\hat{\Omega}_{n}=\tilde{\Omega}_{n}= \begin{cases}R / R y x & n=0 \\ R / R(1-q) y x & n>0\end{cases}
$$

and direct computation yields

$$
\mathrm{H}_{2}(\Omega, b, B) \cong k,
$$

with basis given by the class of $(0, y+R y x)$, while in $\mathrm{H}_{2}(\hat{\Omega}, \hat{b}, \hat{B})$ and $\mathrm{H}_{2}(\Omega, b, d)$ there is an additional generator represented by $((1-q) y, 0)$,

$$
\mathrm{H}_{2}(\hat{\Omega}, \hat{b}, \hat{B}) \cong \mathrm{H}_{2}(\Omega, b, d) \cong k \oplus k / I \cong \mathrm{H}_{2}(\Omega, b, B) \oplus \mathrm{H}_{1}(\mathrm{im} \xi, b, B) .
$$

Note also that $\operatorname{ker} \pi_{2}=0$ here, so the first isomorphism above is canonical in this example.

Example 2.20. Our final example demonstrates that $\operatorname{ker} \pi$ can be nontrivial. To see this, consider the mixed complex

$$
\Omega_{n}:= \begin{cases}\mathbb{C} & n=0,1,2 \\ 0 & n>2\end{cases}
$$

with (co)boundary maps

$$
b_{n}:=\left\{\begin{array}{ll}
\text { id } & n=1, \\
0 & n \neq 1,
\end{array} \quad d_{n}:= \begin{cases}\text { id } & n=1 \\
0 & n \neq 1\end{cases}\right.
$$

Taking $c_{n}:=1$ for all $n$, we obtain

$$
\mathrm{T}_{n}(\tilde{\Omega})= \begin{cases}\mathbb{C} & n=0 \text { or } n=2 k+1, \\ \mathbb{C} \oplus \mathbb{C} & n=2 k+2 .\end{cases}
$$

Here, $(0,1)=\tilde{b}(1) \in \operatorname{ker} \tilde{B} \cap \operatorname{im} \tilde{b} \subset \mathrm{T}_{2}(\tilde{\Omega})$ generates $\operatorname{ker} \pi_{2} \cong \mathbb{C}$, and we have that $\operatorname{im} \tilde{b} \cap \operatorname{im}(\tilde{b}+\tilde{B})=0$.

\section{References}

[1] J.-L. Brylinski, A differential complex for Poisson manifolds, J. Differential Geom. 28 (1988), no. 1, 93-114. MR950556 (89m:58006)

[2] A. Connes, Cohomologie cyclique et foncteurs Ext ${ }^{n}$, C. R. Acad. Sci. Paris Sér. I Math. 296 (1983), no. 23, 953-958 (in French, with English summary). MR777584 (86d:18007)

[3] _ Noncommutative differential geometry, Publ. Math. Inst. Hautes Études Sci. 62 (1985), 257-360. MR823176 (87i:58162)

[4] A. Connes and H. Moscovici, Hopf algebras, cyclic cohomology and the transverse index theorem, Comm. Math. Phys. 198 (1998), no. 1, 199-246.

[5] M. Crainic, Cyclic cohomology of Hopf algebras, J. Pure Appl. Algebra 166 (2002), no. 1-2, 29-66.

[6] J. Cuntz and D. Quillen, Cyclic homology and nonsingularity, J. Amer. Math. Soc. 8 (1995), no. 2, 373-442, DOI 10.2307/2152822. MR1303030 (96e:19004) 
[7] W.G. Dwyer and D.M. Kan, Normalizing the cyclic modules of Connes, Comment. Math. Helv. 60 (1985), no. 4, 582-600, DOI 10.1007/BF02567433. MR826872 (88d:18009)

[8] _ Three homotopy theories for cyclic modules, Proceedings of the Northwestern Conference on Cohomology of Groups (Evanston, Ill., 1985), 1987, pp. 165-175, DOI 10.1016/0022-4049(87)90022-3. MR885102 (88f:18022)

[9] T. Hadfield and U. Krähmer, Braided homology of quantum groups, J. K-Theory 4 (2009), no. 2, 299-332.

[10] P.M. Hajac, M. Khalkhali, B. Rangipour, and Y. Sommerhäuser, Hopf-cyclic homology and cohomology with coefficients, C. R. Math. Acad. Sci. Paris 338 (2004), no. 9, 667-672, DOI 10.1016/j.crma.2003.11.036 (in English, with English and French summaries). MR2065371

[11] J. Huebschmann, Lie-Rinehart algebras, Gerstenhaber algebras and BatalinVilkovisky algebras, Ann. Inst. Fourier (Grenoble) 48 (1998), no. 2, 425-440. MR1625610 (99b:17021)

[12] C. Kassel, Cyclic homology, comodules, and mixed complexes, J. Algebra 107 (1987), no. 1, 195-216, DOI 10.1016/0021-8693(87)90086-X. MR883882 (88k: 18019)

[13] J.-L. Koszul, Crochet de Schouten-Nijenhuis et cohomologie, Astérisque Numero Hors Serie (1985), 257-271 (in French). The Mathematical Heritage of Élie Cartan (Lyon, 1984). MR837203 (88m:17013)

[14] J. Kustermans, G.J. Murphy, and L. Tuset, Differential calculi over quantum groups and twisted cyclic cocycles, J. Geom. Phys. 44 (2003), no. 4, 570-594, DOI 10.1016/S0393-0440(02)00115-8. MR1943179

[15] J.-L. Loday, Cyclic Homology, 2nd ed., Grundlehren der Mathematischen Wissenschaften [Fundamental Principles of Mathematical Sciences], vol. 301, Springer-Verlag, Berlin, 1998. Appendix E by María O. Ronco; Chapter 13 by the author in collaboration with Teimuraz Pirashvili. MR1600246 (98h:16014)

[16] J.M. Shapiro, Relations between twisted derivations and twisted cyclic homology, Proc. Amer. Math. Soc. 140 (2012), no. 8, 2647-2651, DOI 10.1090/S0002-99392011-11285-1. MR2910752

Ulrich Krähmer ulrich.kraehmer@tu-dresden.de

TU Dresden, Institut für Geometrie, 01062 Dresden, Germany

Dylan Madden D.Madden@warwick.ac.uk

B0.15, Mathematics Institute, Zeeman Building, University of Warwick, Coventry, United Kingdom, CV4 7AL 